\title{
EVALUACIÓN AGRONÓMICA Y NUTRICIONAL DEL PASTO RYEGRASS PERENNE TETRAPLOIDE (Lolium perenne) PRODUCIDO EN LECHERÍAS DE LAS ZONAS ALTAS DE COSTA RICA. II. VALOR NUTRICIONAL
}

\author{
Luis Villalobos ${ }^{1 / *}$, Jorge Ml. Sánchez
}

\begin{abstract}
Palabras clave: Pastos clima templado, Lolium perenne, valor nutricional, contenido energético.
\end{abstract}
Keywords: Temperate climate grasses, Lolium perenne, nutritional value, energy content.

Recibido: 30/11/09

\section{RESUMEN}

Se determinó el valor nutricional del pasto ryegrass perenne tetraploide (Lolium perenne) en 4 fincas comerciales de ganado lechero ubicadas en Chicuá de Oreamuno (latitud 0959', longitud $83^{\circ} 52^{\prime}$, altitud $3090 \mathrm{msnm}$ ), provincia de Cartago. Las muestras se tomaron cada 2 meses a una altura de cosecha de $10 \mathrm{~cm}$ sobre el suelo, simulando el pastoreo que realizan los animales durante un período de un año. La composición nutricional promedio anual fue de 25,21\% PC, 46,26\% FDN, 25,57\% FDA, 3,29\% lignina, $15,40 \%$ CNF y $77,95 \%$ DIVMS y su contenido energético, expresado como TND, ED, EM, $\mathrm{EN}_{\mathrm{L}}(3 \mathrm{X})$ y $\mathrm{EN}_{\mathrm{G}}$, fue $61,95 \%, 2,92,2,45,1,53 \mathrm{y}$ 0,92 Mcal. $\mathrm{kg}^{-1}$ de MS, respectivamente. El valor nutricional del pasto ryegrass perenne varió $(\mathrm{p} \leq 0,05)$ según la época del año y el manejo en finca, especialmente en lo referente al período de recuperación de las pasturas. Este pasto tiene un contenido alto de PC y CNF, que permite a nivel ruminal la producción de proteína microbial, la cual es la mejor proteína que puede consumir un rumiante. En general, el valor nutricional del pasto Ryegrass perenne producido a altitudes superiores a los 2500 msnm en Costa Rica es similar, al producido en zonas de clima templado de donde es originario.
Aceptado: 26/02/10

\begin{abstract}
Agronomic and nutritional evaluation of perennial tetraploid ryegrass-based pastures (Lolium perenne) in dairy farms on the highlands of Costa Rica. II. Nutritional Value. The nutritional value of perennial tetraploid ryegrass (Lolium perenne)-based pastures was evaluated in four commercial dairy farms in the district of Chicuá, Oreamuno, Cartago (latitude $09^{\circ} 59^{\prime}$, longitude $83^{\circ} 52^{\prime}, 3090 \mathrm{~m}$ of altitude). Samples were taken every 2 months during a one year period in the paddocks ready to be grazed, simulating DM consumption by lactating cows. Average nutritional value was $25.21 \% \mathrm{CP}, 46.26 \%$ NDF, 25.57\% ADF, 3.29\% lignin, 15.40\% NFC, $77.95 \%$ IVDMD on dry matter basis. Estimated energy content, expressed as TDN, DE, ME, $\mathrm{NE}_{\mathrm{L}}(3 \mathrm{X})$ and $\mathrm{NE}_{\mathrm{G}}$, was $61.95 \%, 2.92,2.45,1.53$ y $0.92 \mathrm{Mcal}^{\mathrm{kg}} \mathrm{kg}^{-1}$ of DM, respectively. Nutritional value of perennial ryegrass pastures is affected by season and pasture management, specially as regards the regrowth period of the paddocks. These pastures have a high CP and NFC content, which allows microbial protein production, but also requires supplements rich in NFC to maximize utilization of these nutrients. In general, nutritional value of this pasture grown at altitudes higher than $2500 \mathrm{~m}$ in Costa Rica is similar to the same grass species grown in temperate climate regions, where it is native.
\end{abstract}

Centro de Investigaciones en Nutrición Animal y Escuela de Zootecnia, Universidad de Costa Rica. San José, Costa Rica. 


\section{INTRODUCCIÓN}

La rentabilidad de la producción de leche en las zonas tropicales se sustenta en las prácticas de manejo de los pastos, los cuales constituyen la fuente de nutrimentos más económica que puede consumir un rumiante. El costo de producción de las gramíneas forrajeras es menos susceptible a la volatilidad de los precios de alimentos balanceados que han caracterizado al mercado internacional en el inicio del siglo XXI (Mercanet 2008), mientras que debido al uso de ciertos cereales y leguminosas para la producción de biocombustibles, los forrajes constituyen alrededor del 3,5\% de los costos de la alimentación del ganado lechero en Costa Rica, costos que a su vez representan aproximadamente el $41 \%$ de los costos totales de producción (Solano y León 2005).

La utilización eficiente de los pastos se fundamenta en 2 aspectos: el manejo del pasto y la suplementación estratégica de este alimento a los animales. Para el manejo de la planta se debe considerar su fenología para cosecharla en el momento en que tiene su mejor contenido de nutrimentos y a la vez suficientes reservas de carbohidratos solubles en sus coronas para sobreponerse a la defoliación causada por el pastoreo y continuar con un nuevo ciclo de crecimiento (Fulkerson y Donaghy 2001). Por su parte, las prácticas de alimentación del ganado bovino deben considerar el aporte de nutrimentos del forraje, para suplementar aquellos nutrimentos que el animal requiere según su estado fisiológico o productivo pero que el pasto no suplió en las cantidades requeridas para promover una buena fermentación ruminal, uso de nutrimentos y en general, una buena nutrición y respuesta productiva del animal (Bargo et al. 2002).

Existen diferentes especies de pastos $\mathrm{C}_{3}$ de clima templado como ryegrass, festucas y Phalaris que han sido introducidas en ciertas regiones del trópico donde la altitud, temperatura, irradiación solar y horas luz permiten su adaptación (Neal et al. 2007). Sin embargo, la literatura universal es escasa en información sobre el valor nutricional de estas gramíneas en zonas tropicales, evaluadas a la luz de las metodologías de uso actual (NRC 2001).

La producción de leche de las vacas que consumen especies de gramíneas $\mathrm{C}_{4}$ del subtrópico y trópico es menor a la de animales que consumen especies $\mathrm{C}_{3}$ de clima templado, debido a que consumen menos energía digestible según Van Soest y Giner-Chavez (1994). Lo anterior se debe a que los pastos tropicales y subtropicales contienen más fibra detergente neutro, lo cual hace que los animales consuman menos materia seca y que la misma aporte una cantidad menor de energía (NRC 2001). La utilización de subproductos de la agroindustria de las frutas tropicales tales como piña, banano y cítricos (Sánchez y Herrera 2006) y de la molinería de granos como el trigo y arroz (Araya 2002) en la alimentación del ganado bovino puede aportar energía en su forma de carbohidratos de fácil disponibilidad, la cual es la forma de energía más limitante en los sistemas de producción de leche o carne que se basan en el pastoreo.

Según Donaghy y Fulkerson (2001) cuando se suplementan los bovinos debe considerarse que la respuesta animal depende del nivel de sustitución del pasto y del efecto que dichos suplementos tengan sobre la fermentación del forraje a nivel ruminal. De este modo, si las vacas lactantes tienen a disposición pasto de buena calidad y se suplementan abundantemente, la utilización de la pastura disminuye y por consiguiente la productividad de la actividad pecuaria es menor. Asimismo, la suplementación excesiva y no balanceada puede causar desórdenes metabólicos como cetosis, que deprimen la salud y comportamiento productivo de los animales (Dann et al. 2005).

Dentro de los componentes de la dieta, el forraje es el más variable en cuanto a su valor nutricional, ya que éste varía según sea su estado de madurez a la cosecha (Hannaway et al. 1999). Fulkerson et al. (1998) indican que para que los forrajes sean de buen valor nutricional sus contenidos de carbohidratos estructurales 
deben ser bajos y degradables, el contenido de carbohidratos solubles altos y en balance con los aminoácidos, la proteína debe ser de baja degradabilidad ruminal que implica mayor proteína de sobrepaso, un contenido de lípidos superior al $5 \%$ de la materia seca y niveles de taninos inferiores a $6 \%$, condiciones que no son fáciles de lograr.

El objetivo de la presente investigación fue estimar el valor nutricional del pasto ryegrass perenne durante un ciclo climático de un año, basado en metodologías de análisis bromatológico vigentes, producido en fincas dedicadas a la producción de ganado lechero ubicadas en la zona aledaña al volcán Irazú en Cartago, clasificada como zona de bosque pluvial tropical de montano.

\section{MATERIALES Y MÉTODOS}

En Villalobos y Sánchez (2010a) se describe la zona, las fincas evaluadas y las características climáticas de la zona donde se realizó la presente investigación.

Se tomó un total de 48 muestras (2 muestras por finca por muestreo bimensual) para estimar el valor nutricional del pasto ryegrass perenne a una altura de cosecha de $10 \mathrm{~cm}$ sobre el suelo, simulando el pastoreo que realizan los animales con base en observaciones realizadas previas al muestreo. Se determinó el contenido de materia seca (MS) y proteína cruda (PC) (AOAC 1990), extracto etéreo (EE) y cenizas (AOAC 2000), las fracciones de la pared celular (FDN, FDA, hemicelulosa, celulosa, y lignina) (Van Soest y Robertson 1985), el contenido de carbohidratos no fibrosos (Van Soest et al. 1991) y la digestibilidad "in vitro" de la materia seca (DIVMS) (Van Soest y Robertson 1985). La energía en sus diferentes expresiones $\left(\mathrm{ED}, \mathrm{EM}, \mathrm{EN}_{\mathrm{L}}\right.$ $3 \mathrm{X} \mathrm{y} \mathrm{EN}_{\mathrm{G}}$ ) se estimó al emplear las metodologías descritas en el NRC (2001).

La información obtenida para las variables de calidad nutricional se sometió al siguiente modelo con el objeto de buscar efectos significativos $(\mathrm{p} \leq 0,05)$ :
Variable analizada $=\mu+$ Mes $_{i}+$ Finca $_{j}+$ Mes*Finca $+_{\mathrm{ij}}$ $\mu=$ media de las variables de valor nutricional Mes $=$ i-ésimo efecto de los meses

Finca $=\mathrm{j}$-ésimo efecto de las fincas

Mes* Finca $=$ interacción meses y fincas $\mathrm{e}=$ residuales del modelo.

Se aplicó la prueba de Duncan para establecer diferencias significativas $(p \leq 0,05)$ entre medias en los diferentes meses y fincas para todas las variables.

\section{RESULTADOS Y DISCUSIÓN}

\section{Componentes intracelulares}

El contenido de MS del pasto ryegrass perenne varió significativamente según el mes del año $(\mathrm{p} \leq 0,0001)$ debido a las variaciones climatológicas en la zona donde se ubican las fincas estudiadas; conforme se incrementa la precipitación el pasto es más húmedo y su contenido de MS se reduce (Cuadro 1). Al aumentar el contenido de humedad de forma significativa $(\mathrm{p} \leq 0,05)$ durante el período de julio a diciembre en el pasto ryegrass, los productores deben ofrecer fuentes de fibra que ayuden a mantener un consumo de MS, disminuir la incidencia de acidosis ruminal e incrementar el aprovechamiento que hacen los animales del alimento ingerido (Aikman et al. 2008).

El contenido de PC promedio fue de $25,21 \%$, valor superior al encontrado por Andrade (2006) en el pasto kikuyo (22,38\%). El porcentaje indicado inicialmente difirió en forma significativa por el efecto de la finca $(\mathrm{p}=0,0003)$ y la interacción mes*finca $(\mathrm{p}=0,0229)$ debido a que las fincas, a pesar de estar en un área cercana a los $20 \mathrm{~km}^{2}$ entre sí, tienen diferentes microclimas que afectan la fisiología de la planta y sus períodos de recuperación (Cuadro 2). El contenido de PC en el pasto ryegrass es muy alto y una alta proporción del mismo es degradable en rumen, por lo que es necesario que la dieta de los animales 
Cuadro 1. Contenido de materia seca, proteína cruda y carbohidratos no fibrosos del pasto ryegrass perenne producido en el distrito de Chicuá, Oreamuno, Cartago. 2005.

\begin{tabular}{lccc}
\hline Meses & Materia seca (\%) & Proteína cruda (\% MS) & CNF(\% MS) \\
\hline Ene-Feb & $18,54^{\mathrm{a}}$ & 25,73 & $10,62^{\mathrm{d}}$ \\
Mar-Abr & $19,02^{\mathrm{a}}$ & 24,54 & $13,64^{\mathrm{cd}}$ \\
May-Jun & $16,04^{\mathrm{b}}$ & 24,42 & $15,92^{\mathrm{bc}}$ \\
Jul-Ago & $14,46^{\mathrm{c}}$ & 24,32 & $20,68^{\mathrm{a}}$ \\
Set-Oct & $14,32^{\mathrm{c}}$ & 26,30 & $13,85^{\mathrm{cd}}$ \\
Nov-Dic & $13,13^{\mathrm{c}}$ & 25,96 & $17,68^{\mathrm{ab}}$ \\
Promedio & $15,92^{1}$ & 25,21 & 15,40 \\
\hline
\end{tabular}

a, b, c medias con letras diferentes en una misma columna difieren entre sí, según la prueba de Duncan $(\mathrm{p} \leq 0,05)$.

1. Valores corresponden al promedio de 48 muestras.

Cuadro 2. Contenido de materia seca, proteína cruda y carbohidratos no fibrosos del pasto ryegrass perenne en 4 fincas productoras de ganado lechero en el distrito de Chicuá, Oreamuno, Cartago.

\begin{tabular}{ccccc}
\hline Finca & Recuperación (días) & Materia seca (\%) & Proteína cruda (\%) & CNF (\%) \\
\hline 1 & 45 & $15,41^{\mathrm{ab}}$ & $24,67^{\mathrm{b}}$ & $16,00^{\mathrm{a}}$ \\
2 & 35 & $16,52^{\mathrm{a}}$ & $22,88^{\mathrm{c}}$ & $16,70^{\mathrm{a}}$ \\
3 & 35 & $15,20^{\mathrm{b}}$ & $26,52^{\mathrm{a}}$ & $11,52^{\mathrm{b}}$ \\
4 & 32 & $16,52^{\mathrm{a}}$ & $26,78^{\mathrm{a}}$ & $17,37^{\mathrm{a}}$ \\
Promedio $^{1}$ & 36,75 & 15,92 & 25,21 & 15,40 \\
\hline
\end{tabular}

a, b, c medias con letras diferentes dentro de una misma columna difieren entre sí, según la prueba de Duncan ( $\mathrm{p} \leq 0,05)$.

1. Valores corresponden al promedio de 48 muestras.

que consumen ryegrass sea balanceada y considere su contenido de CNF (Fulkerson et al. 1998).

Según Hollmann et al. (2008) las entradas y salidas de nutrientes en los suelos de las fincas de ganado de leche, principalmente $\mathrm{N}$ y $\mathrm{P}$ generan desbalances en los sistemas productivos que son difíciles de estimar. En el caso de las lecherías intensivas en Costa Rica se utilizan de forma rutinaria los fertilizantes inorgánicos y muy poco los de tipo orgánico; sin embargo, por regulaciones de tipo ambiental muchas fincas utilizan las aguas de lavado de las salas de ordeño, en los sistemas de pastoreo, lo cual podría incrementar los niveles de nitrógeno soluble en la fracción proteica de los forrajes (Van Soest y Robertson 1985).

Las fincas evaluadas normalmente tienen programas de fertilización con dosis superiores a los $200 \mathrm{~kg} \cdot \mathrm{ha}^{-1} \cdot \mathrm{año}^{-1}$ de $\mathrm{N}$ donde a lo largo del año se utilizan diversos productos que consideran desde fórmulas completas hasta urea en las épocas más lluviosas. Debido a esto es que el contenido de PC del pasto ryegrass es alto e inclusivo además de excesivo para animales de bajo a mediano potencial productivo (NRC 2001). 
El contenido promedio de extracto etéreo del pasto ryegrass perenne fue de $2,61 \%$ y difirió en forma altamente significativa por el efecto del mes evaluado $(\mathrm{p} \leq 0,0001)$. Asimismo, el contenido de cenizas fue de $9,96 \%$ y es influenciado de forma significativa por el mes $(\mathrm{p}=0,0239)$, la finca ( $\mathrm{p}=0,0011)$ y la interacción mes*finca $(\mathrm{P}=0,0149)$. Normalmente el contenido de cenizas es afectado por la movilización de nutrimentos minerales en el suelo en relación con la precipitación a lo largo del año. Según Bertsch (1998) la absorción de nutrientes de una planta varía de acuerdo con su etapa fenológica, lo cual es determinante en la planificación de los programas de fertilización; asimismo al iniciarse la época de lluvias se da un fenómeno denominado fluctuación estacional de nitratos, el cual hace que al darse un movimiento más activo de los minerales en el suelo, se refleje de igual forma en el contenido mineral de la planta. Acosta (1995) considera que es importante tener presente el efecto que genera la fertilización nitrogenada no solo sobre el rendimiento de un pasto y su producción de área foliar, sino también el efecto que tiene en la absorción de otros minerales como $\mathrm{Ca}, \mathrm{Mg}$ y $\mathrm{K}$.

El contenido de carbohidratos no fibrosos (CNF) del pasto ryegrass perenne fue de 15,40\% y su contenido fue influenciado de forma altamente significativa según el mes evaluado $(\mathrm{p} \leq 0,0001)$ y de forma significativa por la finca $(\mathrm{p}=0,0004) \mathrm{y}$ la interacción mes*finca $(\mathrm{p}=0,0471)$ (Cuadro $1 \mathrm{y}$ 2); lo que indica que el contenido de CNF tiene una relación estrecha con el clima de la zona, pues fluctúa durante el año con incrementos al inicio y final de la época lluviosa. El efecto del manejo de los pastos sobre esta variable se notó principalmente en la finca 4 , la cual tiene un período de recuperación del pasto menor y a su vez tuvo el contenido de CNF mayor.

Existe una relación estrecha entre la acumulación de carbohidratos hidrosolubles (CHOS) y la temperatura ambiental, Fulkerson y Donaghy (2001) encontraron bajo condiciones ambientales similares con temperatura de $6^{\circ} \mathrm{C}$ por la noche una concentración de carbohidratos de $17,5 \%$ de MS, comparado con $9 \%$ en plantas mantenidas a $12^{\circ} \mathrm{C}$. La acumulación de carbohidratos se debe a la reducción en la tasa respiratoria bajo las condiciones más frías de la noche, o bien durante las épocas del año con las temperaturas más bajas. Este comportamiento tiene un efecto mayor en la disminución de carbohidratos durante la noche ya que durante el día, cuando aumenta la temperatura, la tasa fotosintética y respiratoria también se incrementan. Las cantidades mayores de CNF en los pastos de las fincas 1 y 2 con respecto a los de la finca 3 puede deberse al efecto antes citado, ya que ambas fincas están a una altitud mayor y por lo tanto expuestas a temperaturas menores.

Andrade (2006) encontró un contenido promedio de CNF de 9,00\% en el pasto kikuyo, lo que indica que el pasto ryegrass perenne es más digestible debido a su contenido mayor de dicha fracción. Asimismo, las cantidades altas de CNF en los pastos son una ventaja ya que permiten el aprovechamiento eficiente de la proteína degradable en el rumen, al ser liberados casi en su totalidad, de las células de las plantas después de un tiempo promedio de permanencia de 1,5 $\mathrm{h}$ en el rumen (Boudon et al. 2002). De acuerdo con Zhu et al. (1999) las pasturas frecuentemente son altas en proteína soluble, razón por la cual los alimentos balanceados que se usan en la alimentación del ganado bovino deben aportar principalmente energía para la síntesis microbial en el rumen y la síntesis de proteína láctea, de no ser así, la proteína soluble y degradable del forraje debe ser excretada como urea por medio de la orina.

Cuando los microorganismos del rumen cuentan con los sustratos adecuados (proteína degradable y carbohidratos solubles) se sintetiza una alta producción de proteína microbial, esto explica parcialmente los niveles de producción de leche altos que se pueden lograr a partir del pasto ryegrass (11 kg.animal ${ }^{-1}$.día ${ }^{-1}$ aproximadamente) con respecto a los niveles de producción láctea de animales que consumen pastos $\mathrm{C}_{4}(7 \mathrm{a} 9 \mathrm{~kg})$.

Una de las principales limitantes de los pastos tropicales es su contenido bajo de energía en forma de carbohidratos solubles, lo cual no permite un uso eficiente de la proteína degradable 
en el rumen (Van Soest y Giner-Chávez 1994). La relación entre nitrógeno y carbohidratos hidrosolubles o fácilmente fermentables en el rumen se considera de importancia para una actividad microbial eficiente en el rumen y llega a ser el principal factor limitante para la producción de leche. Esta relación puede oscilar de 0,8 a 1 en el estado de una hoja a 0,16 a 2 en el estado de 3 hojas (Donaghy y Fulkerson 2001). Los niveles de PC en pasturas de ryegrass en estado vegetativo son normalmente muy altos para las vacas lecheras. Por otra parte se destaca que en las etapas tempranas del rebrote una proporción alta del nitrógeno se encuentra en forma de nitratos $\left(\mathrm{NO}_{3}\right)$ debido a esto, debe procurarse que los animales no ingresen a potreros con pastos con un período de recuperación corto a pesar de que parezca estar en una condición adecuada para su utilización (Fulkerson y Lowe 2002).

\section{Componentes de la pared celular}

Los contenidos de fibra detergente neutro (FDN) y fibra detergente ácida (FDA) se utilizan como indicadores de la calidad del forraje. Los valores bajos de FDN y FDA pueden favorecer un mayor consumo de MS y por lo tanto una mayor producción de leche, debido a que ambos son indicadores del llenado físico del animal y de la calidad de la fibra consumida. En los sistemas de pastoreo se busca estimular el consumo de MS proveniente del pasto, pues así se asegura que el rumen tenga una condición adecuada para favorecer los procesos de fermentación, sin embargo la FDN del pasto debe tener una calidad adecuada de tal forma que el llenado físico sea lento para no comprometer el consumo (Aikman et al. 2008).

El contenido de FDN promedio del pasto ryegrass fue de $49,76 \%$ y es influenciado de forma altamente significativa $(\mathrm{p} \leq 0,0001)$ según el mes evaluado; dicho valor es menor al encontrado por Andrade (2006) en el pasto kikuyo (58\% de la MS) (Cuadro 3). Idealmente un forraje no debe poseer contenidos altos de FDN pues el llenado físico limita el consumo y a nivel ruminal debe invertirse más energía en degradar la pared celular para liberar sus componentes intracelulares (Firkins 2005). El contenido de FDN encontrado en el pasto ryegrass perenne se encuentra muy cercano al reportado por las tablas del NRC (2001) para pastos de clima templado, manejados de forma intensiva $(45,8 \%$ de la MS).

El contenido de FDA promedio para el pasto ryegrass perenne fue de 25,57\%; dicha variable se relaciona negativamente con la digestibilidad y el aporte de energía de un pasto. El contenido de FDA fue afectado según el mes $(\mathrm{p}=0,0132)$, la finca $(\mathrm{p}=0,0013)$ y la interacción

Cuadro 3. Contenido de los componentes de la pared celular del pasto ryegrass perenne producido en el distrito de Chicuá, Oreamuno, Cartago.

\begin{tabular}{lccccc}
\hline Meses & $\begin{array}{c}\text { FDN } \\
(\%)\end{array}$ & $\begin{array}{c}\text { FDA } \\
(\%)\end{array}$ & $\begin{array}{c}\text { Hemicel } \\
(\%)\end{array}$ & $\begin{array}{c}\text { Cel } \\
(\%)\end{array}$ & $\begin{array}{c}\text { Lignina } \\
(\%)\end{array}$ \\
\hline Ene-Feb & $58,56^{\mathrm{a}}$ & $27,33^{\mathrm{a}}$ & $31,24^{\mathrm{a}}$ & $22,60^{\mathrm{a}}$ & $2,41^{\mathrm{b}}$ \\
Mar-Abr & $54,97^{\mathrm{a}}$ & $26,27^{\mathrm{ab}}$ & $28,69^{\mathrm{a}}$ & $21,01^{\mathrm{ab}}$ & $3,74^{\mathrm{a}}$ \\
May-Jun & $48,93^{\mathrm{b}}$ & $26,29^{\mathrm{ab}}$ & $22,64^{\mathrm{b}}$ & $20,75^{\mathrm{bc}}$ & $3,17^{\mathrm{ab}}$ \\
Jul-Ago & $44,83^{\mathrm{b}}$ & $24,59^{\mathrm{bc}}$ & $20,24^{\mathrm{b}}$ & $19,17^{\mathrm{bc}}$ & $3,97^{\mathrm{a}}$ \\
Set-Oct & $47,51^{\mathrm{b}}$ & $25,55^{\mathrm{abc}}$ & $21,95^{\mathrm{b}}$ & $19,92^{\mathrm{bc}}$ & $3,40^{\mathrm{a}}$ \\
Nov-Dic & $43,77^{\mathrm{b}}$ & $23,39^{\mathrm{c}}$ & $20,39^{\mathrm{b}}$ & $19,05^{\mathrm{c}}$ & $3,06^{\mathrm{ab}}$ \\
Promedio & $49,76^{1}$ & $25,57^{-}$ & 24,19 & $20,42^{2}$ & 3,29 \\
\hline
\end{tabular}

a, b, c medias con letras diferentes dentro de una misma columna difieren entre sí, según la prueba de Duncan $(\mathrm{p} \leq 0,05)$.

1. Valores corresponden al promedio de 48 muestras. 
mes*finca ( $\mathrm{p}=0,0253)$ (Cuadro 3). La finca 4, en donde el período de recuperación fue menor, tuvo el contenido menor de FDA, lo que indica que es un pasto más aprovechable por el animal y que le da mayor energía.

El contenido promedio de hemicelulosa del pasto ryegrass perenne a lo largo del año fue de $24,19 \%$ y se fue afectado en forma altamente significativa por el mes $(\mathrm{p} \leq 0,0001)$. El contenido de celulosa tuvo un promedio de $20,42 \%$ y difirió significativamente entre meses $(\mathrm{p}=0,0024)$ y fincas $(p=0,0329)$ La interacción mes*finca también fue altamente significativa $(\mathrm{p}=0,0039)$. Debido a que el contenido de hemicelulosa fue siempre superior al de la celulosa, el pasto ryegrass es muy digestible, ya que la hemicelulosa es más accesible que la celulosa a los procesos enzimáticos de los microorganismos del rumen (Pinos et al. 2002) (Cuadro 3).

El contenido de lignina promedio fue de $3,29 \%$ y fue influenciado significativamente por el mes $(\mathrm{p}=0,0163)$, la finca $(\mathrm{p} \leq 0,0006)$ y la interacción mes*finca $(\mathrm{p} \leq 0,0001)$. La finca 4 mostró el contenido menor de lignina. A pesar de que el contenido de lignina del pasto ryegrass es ligeramente mayor que el encontrado por Andrade (2006) en el pasto kikuyo (2,67\%), éste último tiene un contenido de FDN mayor, lo cual permite que el pasto ryegrass sea más digestible. De acuerdo con Van Soest y Giner-Chavez (1994). Por otra parte se identificó que el clima frío es el factor ambiental primario responsable de una síntesis menor de lignina, lo cual coincide con la época seca de la zona estudiada en donde Retana (2006) reporta las temperaturas mínimas más bajas del año llegando a valores cercanos a $0^{\circ} \mathrm{C}$, lo que permite el fenómeno de cristalización del rocío sobre las hojas del pasto. Para pastos de clima templado el NRC (2001) reporta valores de lignina de $2,1 \%$, el cual es inferior al ryegrass perenne analizado; sin embargo, esto varía durante el año y entre fincas, llegando a valores de hasta 2,4\% en los meses más fríos (Cuadro 3).

\section{Digestibilidad in vitro de la materia seca (DIVMS)}

La DIVMS del pasto ryegrass fue influenciado en forma altamente significativa por el mes $(\mathrm{p} \leq 0,0001)$, las fincas $(\mathrm{p}=0,0145)$ y la interacción mes*finca $(p=0,0060)$. Las variaciones en la DIVMS del pasto ryegrass perenne a lo largo del año mostraron una relación inversa con respecto al contenido de pared celular (Cuadro 4). La pared celular limita el acceso de los microorganismos del rumen al contenido celular, el cual es de acuerdo con Boudon et al. (2002) alrededor del $50 \%$ de la MS en pastos de clima templado.

Cuadro 4. Digestibilidad in vitro y contenido de energía estimada del pasto ryegrass perenne durante el año de estudio en el distrito de Chicuá, Oreamuno, Cartago.

\begin{tabular}{|c|c|c|c|c|c|c|}
\hline Meses & $\begin{array}{l}\text { DIVMS } \\
(\%)\end{array}$ & $\begin{array}{l}\text { TND } \\
(\%)\end{array}$ & $\begin{array}{c}\text { ED } \\
\left.\text { (Mcal.kg }{ }^{-1} \text { de } \mathrm{MS}\right)\end{array}$ & $\begin{array}{c}\text { EM } \\
\text { (Mcal. } \mathrm{kg}^{-1} \text { de MS) }\end{array}$ & $\begin{array}{c}\mathrm{EN}_{\mathrm{L}} 3 \mathrm{X} \\
\text { (Mcal.kg }{ }^{-1} \text { de MS) }\end{array}$ & $\begin{array}{c}\mathrm{EN}_{\mathrm{G}} \\
\text { (Mcal.kg-1 de MS) }\end{array}$ \\
\hline Ene-Feb & $76,49^{\mathrm{b}}$ & $59,88^{\mathrm{b}}$ & $2,83^{\mathrm{b}}$ & $2,43^{b}$ & $1,51^{\mathrm{b}}$ & $0,88^{b}$ \\
\hline Mar-Abr & $71,49^{c}$ & $60,17^{\mathrm{b}}$ & $2,83^{b}$ & $2,38^{\mathrm{b}}$ & $1,48^{\mathrm{b}}$ & $0,85^{\mathrm{b}}$ \\
\hline May-Jun & $75,60^{\mathrm{b}}$ & $62,21^{a b}$ & $2,92^{a b}$ & $2,44^{a b}$ & 1,53 ab & $0,92^{a b}$ \\
\hline Jul-Ago & $75,75^{\mathrm{b}}$ & 62,67 ab & $2,94^{a b}$ & $2,45^{a b}$ & $1,53^{a b}$ & 0,93 ab \\
\hline Set-Oct & $78,71^{b}$ & $62,41^{a b}$ & $2,95 \mathrm{ab}$ & $2,46^{\mathrm{ab}}$ & $1,54^{a b}$ & $0,94^{a b}$ \\
\hline Nov-Dic & $89,65^{\text {a }}$ & $64,39^{\text {a }}$ & $3,04^{\mathrm{a}}$ & $2,51^{\mathrm{a}}$ & $1,58^{\mathrm{a}}$ & $0,99^{\mathrm{a}}$ \\
\hline Promedio $^{1}$ & 77,95 & 61,95 & 2,92 & 2,45 & 1,53 & 0,92 \\
\hline
\end{tabular}

a, b, c medias con letras diferentes dentro de una misma columna difieren entre sí, según la prueba de Duncan $(\mathrm{p} \leq 0,05)$.

1. Valores corresponden al promedio de 48 muestras. 
Dentro de los componentes intracelulares se considera que el $60 \%$ de los azúcares libres y el $\mathrm{K}$ y el $30 \%$ de la clorofila son liberados en el momento de la ingesta (Boudon et al. 2002).

Normalmente los pastos de clima templado acumulan reservas de carbohidratos para sobrevivir durante las épocas de temperaturas bajas, sin embargo en condiciones tropicales de altura no se presentan cambios de estación tan marcados que hagan que el crecimiento del pasto se detenga, por lo que el forraje mantiene un nivel de reservas de CNF sin llegar a reproducirse (Van Soest y Giner-Chavez 1994). Andrade (2006) encontró para el pasto kikuyo un promedio de DIVMS de $65,43 \%$, llegando a valores máximos de $69,75 \%$. Lo anterior muestra una diferencia de más de 10 unidades porcentuales de digestibilidad por debajo del promedio obtenido en el pasto ryegrass debido a que éste último tiene un contenido de FDN menor con respecto al pasto kikuyo, lo cual le permite tener un contenido de componentes intracelulares mayor.

\section{Contenido de energía estimada}

El contenido de total de nutrientes digestibles totales (TND) tuvo un efecto significativo del mes $(p=0,0197)$, la finca de procedencia del pasto $(\mathrm{p}=0,0012)$ y la interacción mes*finca $(\mathrm{p}=0,0007)$. El TND siguió la misma tendencia mostrada por la DIVMS al aumentar conforme disminuyó el contenido de FDN hacia final del año y ser menor en la época seca. En el pasto kikuyo se ha estimado valores de TND de $63,72 \%$, que es muy cercano al encontrado en el ryegrass perenne en la zona de Chicuá y además presenta un comportamiento similar al disminuir durante la época seca (Andrade 2006) (Cuadro 4) El NRC (2001) reporta para pastos de clima templado manejados intensivamente valores de TND en promedio de $66,6 \%$, lo cual es muy cercano a lo encontrado en el ryegrass perenne.

$\mathrm{La} \mathrm{ED}, \mathrm{EM}, \mathrm{EN}_{\mathrm{L}}$ y $\mathrm{EN}_{\mathrm{G}}$ mostraron valores promedio de 2,92, 2,45, 1,53 y 0,92 Mcal. $\mathrm{kg}^{-1}$ de MS y mostraron la tendencia mencionada para el TND a lo largo del año y para la finca con período de recuperación menor. Con base en esto, se evidencia que tanto el clima como el manejo de las pasturas en las fincas determinan la calidad de las raciones ofrecidas a los animales en producción.

El contenido de energía de los forrajes es un componente crítico en los sistemas de producción en pastoreo pues se ha estimado que de la energía neta de lactancia $\left(\mathrm{EN}_{\mathrm{L}}\right)$ consumida durante el día, que alrededor de un 25\% depende de la distancia caminada y puede ser empleada para caminar en el potrero y hacia la sala de ordeño (Kolver y Muller 1998; citados por Firkins 2005).

\section{CONCLUSIONES}

El contenido de MS del pasto ryegrass perenne tiene un comportamiento opuesto al del régimen de precipitación; mientras que el contenido de PC se mantiene relativamente constante a lo largo del año. Sin embargo, el período de recuperación de las pasturas afecta el contenido de dicho nutrimento, por lo que por medio del manejo de las pasturas se debe buscar el período adecuado en cada finca para producir un forraje de alta calidad nutricional y acorde con el nivel productivo de los animales en producción.

El contenido de PC del pasto ryegrass perenne expresado en términos de MS, es superior al de muchos alimentos balanceados comerciales por lo que es necesario ofrecer a los animales suplementos que permitan hacer un uso adecuado del nitrógeno, el cual es probable que tenga una alta proporción de proteína degradable y aprovechable por los microorganismos del rumen.

Los componentes de la pared celular disminuyeron durante la época lluviosa y se presentaron variaciones importantes entre fincas debidas al manejo del forraje propio de cada una, esto puede afectar el consumo de MS de los animales con pesos y niveles de producción similares entre fincas. El contenido de CNF varía inversamente con el contenido de FDN, lo cual hace que la DIVMS y el contenido de energía sean mayores al disminuir los componentes de la pared celular. 
El ryegrass perenne es un pasto de clima templado que se puede establecer en condiciones tropicales de altura y cuyo valor nutricional es superior al de los pastos tropicales de altura (>2500 msnm). Asimismo tolera las condiciones de radiación solar y temperaturas bajas que se presentan en dichas zonas.

\section{AGRADECIMIENTOS}

Los autores expresan su agradecimiento a los propietarios de las fincas donde se realizó esta investigación: Sr. Rolando Ferreto, Sra. Gabriela Arrea, Sr. Noré Gómez y Sr. Bernardo Gómez (q.d.D.g.), así como a los trabajadores de las fincas por la colaboración brindada durante el año en que se realizó la investigación.

\section{LITERATURA CITADA}

ACOSTA R. 1995. Fertilizantes y pastoreo rotacional: dos técnicas para alta producción de leche y carne. Cafesa, Costa Rica. 75 p.

AIKMAN P.C., REYNOLDS C.K., BEEVER D.E. 2008. Diet digestibility, rate of passage, and eating and rumination behavior of Jersey and Holstein Cows. Journal of Dairy Science 91:1103-1114.

ANDRADE M. 2006. Evaluación de técnicas de manejo para mejorar la utilización del pasto kikuyo (Pennisetum clandestinum Hochst. Ex Chiov) en la producción de ganado lechero en Costa Rica. Tesis de licenciatura, Universidad de Costa Rica, San José, Costa Rica. $225 \mathrm{p}$.

ARAYA M. 2002. Valor nutritivo de los subproductos de la industrialización del trigo, arroz y pan de devolución utilizados en la alimentación del ganado lechero en Costa Rica. Tesis de licenciatura, Universidad de Costa Rica, San José, Costa Rica. 125 p.

ASSOCIATION OF OFFICIAL AGRICULTURAL CHEMISTS. 2000. Official methods of analysis. 17 ed. Washington, D.C.

ASSOCIATION OF OFFICIAL AGRICULTURAL CHEMISTS. 1990. Official methods of analysis. 15 ed. Washington, D.C. 1008 p.

BARGO F., MULlER L. D., DELAHOY J.E., CASSIDY T.W. 2002. Milk response to concentrate supplementation of high producing dairy cows grazing two pasture allowances. Journal of Dairy Science 85:1777-1792.

BERTSCH F. 1998. La fertilidad de los suelos y su manejo. San José, Costa Rica. Asociación Costarricense de la Ciencia del Suelo. 157 p.

BOUDON A., PEYRAUD J.L., FAVERDIN P. 2002. The release of cell contents of fresh rye-grass (Lolium perenne L.) during digestion in dairy cows: effect of the intracellular constituents, season and stage of maturity. Animal Feed Science and Technology. 97:83-102.

DANN H.M., MORIN D.E., BOLLERO G.A., MURPHY M.R., DRACKLEY J.K. 2005. Prepartum intake, postpartum induction of ketosis, and periparturient disorders affect the metabolic status of dairy cows. Journal of Dairy Science 88:3249-3264.

DONAGHY D., FULKERSON B. 2001. Principles for developing an effective grazing management system for ryegrass-based pastures. Tasmanian Institute of Agricultural Research, Burnie, Tasmania. 10 p.

FIRKINS J. 2005. Forage quality and feeding systems for dairy cattle. In: Curso Actualización en la nutrición del Ganado lechero. RAPCO. Balsa, Atenas, Costa Rica. 13 p.

FULKERSON W.J., DONAGHY D.J. 2001. Plant-soluble carbohydrate reserves and senescence key criteria for developing an effective grazing management system for ryegrass-based pastures: a review. Australian Journal of Experimental Agriculture. 41:261-275.

FULKERSON W.J., LOWE K.F. 2002. Grazing Management. Forages and Pastures. 1142-1149.

FULKERSON W.J., SLACK K., HENNESSY D.W., HOUGH G.M. 1998. Nutrients in ryegrass (Lolium spp), white clover (Trifolium repens) and kikuyu (Pennisetum clandestinum) pastures in relation to season and stage of regrowth in a subtropical environment. Australian Journal of Experimental Agriculture. 38:227-240.

HANNAWAY D., FRANSEN S., CROPPER J. 1999. Annual Ryegrass. Oregon State University,USA Consultado el 11/05/2005. En: http://eesc.orst.edu/AgComWebFile/ EdMat/PNW501.html.

HOLLMANN M., KNOWLTON K.F., HANIGAN M.D 2008. Evaluation of Solids, Nitrogen and Phosphorus Excretion Models for Lactating Dairy Cows. Journal of Dairy Science. 91:1245-1257. 
MERCANET. 2008. Sistema de información e inteligencia de mercados. Boletín $N^{\circ}$. 6. Consejo Nacional de la Producción. 12 p.

NATIONAL RESEARCH COUNCIL. 2001. Nutrient Requirements of Dairy Cattle. $7^{\text {th }}$ rev. Ed. Washington, D.C. National Academy Press. 381 p.

NEAL M., NEAL J., FULKERSON W.J. 2007. Optimal choice of dairy forages in Eastern Australia. Journal of Dairy Science 90:3044-3059.

PINOS J.M., GONZALEZ S.S., MENDOZA E. 2002. Effect of exogenous fibrolytic enzyme on ruminal fermentation and digestibility of alfalfa and ryegrass hay fed to lambs. Journal of Animal Science. 80:3016-3020.

RETANA J. 2006. Climatología de la región del distrito de San Juan de Chicuá y el Volcán Irazú. Informe anual del Instituto Meteorológico Nacional. 3 p.

SÁNCHEZ J.ML., HERRERA C. 2006. Nutritional value of tropical fruit processing by-products. American Dairy Science Association-American Society of Animal Science. Annual Meeting. Minneapolis, Minnesota. Abstract W220.

SÁNCHEZ J.ML., SOTO H. 1999. Estimación de la calidad nutricional de los forrajes del cantón de San Carlos. III. Energía para la producción de leche. Nutrición Animal Tropical 5(1):31-49.
SOLANO P.C., LEÓN H.H. 2005. Análisis de costos de diferentes sistemas de producción de leche en Costa Rica: estudio de casos. Cámara Nacional de Productores de Leche. Presentado en el Congreso Nacional Lechero 2005. 22 p.

VAN SOEST P.J., GINER-CHAVEZ B.I. 1994. Nutritive value of fibrous feeds. IN: Sistemas de Producción de Ganado de carne en el trópico. Balsa, Atenas, Costa Rica. 10 p.

VAN SOEST P.J., ROBERTSON J.B. 1985. Analysis of forages and fibrous feeds. Cornell University. Ithaca, New York. 165 p.

VAN SOEST P.J., ROBERTSON J., LEWIS B. 1991. Methods for dietary fiber, neutral detergent fiber and nonstarch polysaccharides in relation to animal nutrition. Journal of Dairy Science 74:3586-3597.

VILLALOBOS L., SÁNCHEZ J.ML. 2010a. Evaluación agronómica y nutricional del pasto riegrass perenne tetraploide (Lolium perenne) producido en lecherías de las zonas altas de Costa Rica .I. Producción de biomasa y fenología. Agronomía Costarricense 34 (1): 31-42.

ZHU W.Y., KINGSTON-SMITH A.H., TRONCOSO D., MERRY R.J., DAVIES D.R., PICHARD THOMAS H., THEODOROU M.K. 1999. Evidence of a Role for Plant Proteases in the Degradation of Herbage Proteins in the Rumen of Grazing Cattle. Journal of Dairy Science 82:2651-2658. 